\title{
OPTIMAL EXECUTION STRATEGY OF LIQUIDATION
}

\author{
KA WO LAU ${ }^{\mathrm{a}}$, YUE KUEN KWOK ${ }^{\mathrm{b}, 1}$ \\ a Citigroup, Hong Kong, China \\ b Department of Mathematics \\ Hong Kong University of Science and Technology \\ Clear Water Bay, Hong Kong, China
}

\begin{abstract}
Liquidity risks arise from the presence of execution time lags on execution of market orders in trading securities and "quantity" effect (liquidation discount) on security price. In this paper, we consider an investor who is holding a portfolio of stock and cash (in the form of market money account) with the objective to unwind his position on the risky asset so that the expected value of cash at the end of a fixed time horizon is maximized. Assuming that the executive time lags and liquidation discount are deterministic, we construct the numerical algorithms for computing the optimal trading strategy that maximizes the expected terminal value of cash position in the portfolio. We also investigate the probability of meeting the target cash level under different liquidation discount functions.
\end{abstract}

1. Introduction. Liquidity risks are related to the time delay and price effect of execution of sell or buy market orders of an asset in the financial market. An illiquid secondary market may prevent the liquidation or limit the cash that can be generated from the liquidation of the asset. Institutional traders are often not able to raise cash within a time constraint by unwinding the portfolio when the markets have are gradually drying [7].

The competitive market paradigm assumes that the security markets are perfectly elastic and all market orders can be executed instantaneously. Elastic markets mean there is no "quantity effect" on the security price. However, in real market situations, when the market participants detect an unusual high volume of sell order, they would naturally adjust their bids to a lower level. As a result, there is a price discrepancy between the price of security at the time when a large trade order is placed and at the time when the trade order is executed. The difference in these prices is called the liquidation discount. Also, the transaction may take a considerable amount of time to complete so that there are execution time lags in selling a large amount of shares. Assuming that the execution time lags and liquidation discount are deterministic, the optimal liquidation problem is to develop an optimal execution strategy such that a trader can unwind a portfolio position within a fixed time constraint subject to the optimization of certain criteria, like the minimization of the expected shortfall in value.

2000 Mathematics Subject Classification. Primary: 91B28.

Key words and phrases. liquidation discount, optimal execution strategy, execution time lags.

1 CORRESPONDING AUTHOR. 
Bertsimas and Lo [4] consider a similar problem where the trader wants to acquire stocks within a fixed finite time horizon. They define the best execution strategy in the sense that it provides the minimum expected cost of acquiring the stocks. In their trading model, they add an impact premium on the execution price of the trade. Such premium is modeled by a pre-defined price impact function that yields the execution price of an individual trade as a function of the shares trades. In a later paper, Bertsimas et al. [3] develop an effective numerical algorithm for finding the optimal execution strategy of acquisition of a tradeable asset.

Almgren and Chriss [2] extend the work of Bertsimas and Lo [4] in two aspects. First, they split the total price impact into the temporary and permanent components. The temporary impact refers to temporary imbalances in supply and demand caused by a trade order, and the price drop only has impact on this trade. On the contrary, the permanent impact refers to price drop that persists for the whole life of the liquidation period. The temporary and permanent price impacts are both modeled by deterministic functions, taking the average rate of trading as an input parameter. Second, their model also incorporates the uncertainty of trading cost. Under their framework, the optimal execution strategy minimizes the sum of the expectation and variance of the liquidation discount. In a later work, Almgren [1] considers optimal trading strategies with a power law function of the trading rate for liquidation of a large single-asset portfolio that minimize the volatility risk and market impact costs.

Subramanian and Jarrow [8] incorporate both the execution lags and liquidation discounts in their liquidation model. To model the execution lags, they impose a rule to the trader that he is not allowed to place any additional sell order before the previous sell order is completely executed. The waiting time depends on the number of shares in previous sell order, and is represented by a pre-defined deterministic function. In addition, a sell order also generates a permanent price drop whose magnitude depends on the amount of the sell order. The price drop again is modeled by a pre-specified deterministic function. An optimal execution scheme is then developed to maximize the sum of expected utility value gained for each sell order.

Longstaff [7] considers a related problem which he calls an optimal portfolio choice problem. They consider a portfolio consisting of riskless asset and illiquid risky securities. Under uncertainty of the stock price dynamics, an investor then seeks an optimal self-financing rebalance strategy to maximize the expected utility value of his terminal wealth. Longstaff models the execution time lags of the trading order by imposing an upper bound on the number of risky assets that the trader can trade per period. His paper reports an intuitive finding that the investor will choose a lower initial portfolio weight of risky securities in the presence of liquidity constraints. Moreover, his results also show that the percentage price discounts for illiquid stocks can be substantial.

Duffie and Ziegler [5] investigate how common risk measures such as likelihood of insolvency, value at risk and expected tail loss respond to widening bid-ask spreads when positions are liquidated within a short period for maintaining capital ratio. They consider two possible liquidation strategies. The first strategy is to minimize expected transaction cost by selling liquid assets first. However, this would increase the tail losses and the probability of insolvency. An alternative strategy is to sell illiquid asset and keep a cushion of liquid assets, at the expense of increasing the expected transaction costs. 
In this paper, we consider a liquidation model that extends the models of Longstaff [7] and Subramanian and Jarrow [8]. Similar to the work by Subramanian and Jarrow, we model the execution time lags and liquidation discount by deterministic functions. Assuming that the trader's objective is to unwind his position of the risky asset to meet some target cash level at the end of a fixed trading period, we develop an effective numerical algorithm for finding the optimal execution strategy of liquidation that maximizes the expected terminal value of cash position in the portfolio.

This paper is organized as follow. In the next section, we present the formulation of the liquidation model. The assumptions on the price dynamics of the risky asset, execution time lags and liquidation discount are presented. The objective of the optimal liquidation procedure is defined. In Section 3, we present the forward shooting grid algorithms for finding the optimal execution strategy and the probability of meeting a pre-set cash level at the end of the liquidation period. Numerical calculations of the liquidation procedure are presented in Section 4. In particular, we explore the impact of varying parameter values in the liquidation discount function, volatility of asset price and target cash level on the probability of terminal cash position above the target by the liquidation procedure. The last section contains conclusive remarks of our liquidation model on optimal execution strategy of liquidation.

2. Formulation of the liquidation model. Suppose an investor initially holds a portfolio consisting of risky asset and cash (in the form of money market account) and he would like to unwind his position on the risky asset so as to meet some target cash level at the end of the liquidation period. The proceeds from the sale of the risky asset is added to the cash position and the trading is self-financing. The stochastic dynamics of the price of the risky asset $S$ and the money market account $B$ are assumed to be:

$$
\begin{gathered}
\frac{d S}{S}=\mu d t+\sigma d Z, \\
\frac{d B}{B}=r d t,
\end{gathered}
$$

where $\mu$ and $\sigma$ denote the constant drift rate and volatility of $S$, respectively. The riskless interest rate $r$ is assumed to be constant.

2.1. Execution time lags and liquidation discount. Following similar assumptions as adopted by Subramanian and Jarrow [8], we assume that the trader encounters liquidity discount on the sale of the risky asset and time lags in the execution of the selling orders. Both the liquidity discount and execution time lag are defined exogenously. Suppose the trader places a selling order of $s$ units of the risky asset in the market at time $t$, the execution time lag is defined as $\Delta(s)$. This means that the trader cannot make any further sell order within the time interval $(t, t+\Delta(s))$, and the proceeds of the sale are collected at time $t+\Delta(s)$. We assume that $\Delta(s)$ is a deterministic and non-decreasing function in $s$ with $\Delta(0) \geq 0$. The non-decreasing property is consistent with the intuition that larger sales take more time to execute. Furthermore, we assume that a selling order of $s$ units of the risky asset will generate an instantaneous price drop on the asset price as modeled by

$$
S\left(t^{+}\right)=\alpha(s) S(t)
$$

where $0<\alpha(s) \leq 1$, and $S\left(t^{+}\right)$represents the asset price at the moment right after time $t$ at which the sell order is submitted. The factor $\alpha(s)$ represents the price 
discount due to a selling order of $s$ units of risky asset placed in the market. We assume $\alpha(s)$ to be a deterministic, non-negative and non-increasing function in $s$ with $\alpha(0)=1$. In our model, the "quantity" impact on price is assumed to be cumulative and permanent, and the price drop persists for the whole liquidation period.

2.2. Statement of the problem. Subject to execution time lag and liquidation discount, the investor's objective is to seek an optimal execution strategy to unwind his position on the risky asset within a fixed time period $T$ such that the expected terminal value of the cash position is maximized. We adopt the self-financing strategy where there is no additional funds added or withdrawn during the liquidation period, and the proceeds of the sale of the risky asset are added to the cash position.

Let $c_{T}$ denote the cash value held by the investor at time $T$. We would like to maximize the expected value of $c_{T}$ among all possible liquidation strategies. The expected maximum terminal value of cash at time $T$ has dependence on the number of units of risky asset $n$, asset price $S$ and cash level $c$ in the portfolio at time $t, t<T$. Let $U(S, n, c, t)$ denote the functional dependence of the expected maximum value of $c_{T}$ on $S, n, c$ and $t$. Given that the initial portfolio at $t=0$ contains $n_{0}$ units of risky asset, cash amount $c_{0}$ and asset price $S_{0}$, our objective is to compute $U\left(S_{0}, n_{0}, c_{0}, 0\right)$. Let $D$ denote the pre-set target cash level at the end of the liquidation period. We also compute the probability that the pre-set target level is met on the terminal date, that is, $P\left[c_{T} \geq D\right]$.

3. Construction of the forward shooting grid algorithm. First, we construct a trinomial tree to simulate the stochastic movement of the stock price. When we compute the expected terminal value of cash position, the expectation is taken over all scenarios of asset price movement and possible inter-temporal selling of stocks. Upon the sale of certain units of risky asset, the total number of units of risky asset held has to be adjusted, and the proceeds collected are added to the cash position. Such complicated path dependence structure can be handled effectively by the forward shooting grid approach [6].

In the trinomial tree framework, we let $U_{j, \mathbf{F}}^{n}$ denote the numerical approximation of the expected terminal value of cash position maximized among all liquidation strategies. The indices $n$ and $j$ denote the $n^{\text {th }}$ time step and $j$ upward jumps from the initial asset value in the trinomial tree. Let $x$ and $c$ denote the number of units of risky assets and value of cash, respectively, in the prevailing portfolio. These path-dependent state variables are stored in the augmented vector $\mathbf{F}$, and we write

$$
\mathbf{F}=\langle x, c\rangle \text {. }
$$

We discretize the cash variable $c$ so that the value taken on by $c$ are

$$
c(\ell)=c_{0}+\ell \Delta c, \quad \ell=0,1, \cdots, L,
$$

where $c_{0}$ represents the initial cash amount.

Let $V_{j, \mathbf{F}}^{n, s}$ denote the numerical approximation of the maximum expected terminal riskless asset value right after the sell order of $s$ units of shares is placed on the order book. As the trader would examine all feasible selling orders and select a trading strategy that generates the maximum expected terminal value of the cash position, the dynamic programming scheme can be succinctly represented by

$$
U_{j, \mathbf{F}}^{n}=\max _{0 \leq s \leq x}\left[V_{j, \mathbf{F}}^{n, s}\right], \quad \text { if } n \Delta t+\Delta(s) \leq T .
$$


Here, we require $n \Delta t+\Delta(s) \leq T$ since the liquidation process has to be completed by the terminal date $T$. Let $c^{n}$ denote the cash value at time step $n$. We consider the following two cases.

1. Suppose no sell order is placed by the trader (i.e., $s=0$ ), then the cash value at the next time step $c^{n+1}$ is

$$
c^{n+1}=c^{n}(1+r \Delta t) .
$$

In general, $c^{n+1}$ does not fall onto one of the pre-set values in Eq. (4). We use the quadratic interpolation rule to compute the required nodal value from neighboring nodal values. Let $c_{\ell^{\prime}-1}, c_{\ell^{\prime}}$ and $c_{\ell^{\prime}+1}$ be the neighboring nodes of $c^{n+1}$, and let $U_{j^{\prime},\langle x, \tilde{\ell}\rangle}^{n+1}$ denote the interpolated nodal value for cash $c^{n+1}$. The quadratic interpolation procedure is given by

$$
U_{j^{\prime},\langle x, \widetilde{\ell}\rangle}^{n+1}=\alpha_{-1}^{\ell^{\prime}} U_{j^{\prime},\left\langle x, \ell^{\prime}-1\right\rangle}^{n+1}+\alpha_{0}^{\ell^{\prime}} U_{j^{\prime},\left\langle x, \ell^{\prime}\right\rangle}^{n+1}+\alpha_{1}^{\ell^{\prime}} U_{j^{\prime},\left\langle x, \ell^{\prime}+1\right\rangle}^{n+1} .
$$

After the required nodal values have been computed at time level $n+1$, we then use backward induction to proceed to time level $n$. As there is no price impact when $s=0$ so that $S\left(n \Delta t^{+}\right)=S(n \Delta t)$, we then have

$$
\begin{aligned}
V_{j, \mathbf{F}}^{n, 0} & =E\left[U_{j^{\prime},\langle x, \tilde{\ell}\rangle}^{n+1} \mid S(n \Delta t)=S_{j}\right] \\
& =p_{u} U_{j+1,\langle x, \tilde{\ell}\rangle}^{n+1}+p_{m} U_{j,\langle x, \tilde{\ell}\rangle}^{n+1}+p_{d} U_{j-1,\langle x, \tilde{\ell}\rangle}^{n+1}
\end{aligned}
$$

where $p_{u}, p_{m}$ and $p_{d}$ are the respective probabilities of upward jump, zero jump and downward jump in the trinomial tree (see Figure 1).

2. Suppose the trader places a selling order of $s$ units of asset in the market. The proceeds of the trade are collected at time step $n+k$, where $k \Delta t=\Delta(s)$. The cash value $c^{n+k}$ at time step $n+k$ is

$$
c^{n+k}=c^{n}(1+r k \Delta t)+s S((n+k) \Delta t) .
$$

The first term in the above equation represents cash (in the form of money market account) grows at the riskless rate $r$, and the second term represents the amount of newly acquired cash as a result of collecting the proceeds of selling $s$ units of risky asset at time step $n+k$. Again, $c^{n+k}$ in general does not fall onto the pre-set nodal values of cash. We use quadratic interpolation to compute the required nodal values at time step $n+k$. Let the interpolated nodal value for cash $c^{n+k}$ be $U_{j^{\prime},\langle x-s, \tilde{\ell}\rangle}^{n+k}$ (note that the number of units of asset drops to $x-s$ ). When the interpolated nodal values for cash $c^{n+k}$ at time level $n+k$ are available, we then perform usual backward induction to time level $n$. Since there is an instantaneous price drop on the asset price right after the selling order of $s$ units of risky asset is placed, we have $S\left(n \Delta t^{+}\right)=\alpha(s) S(n \Delta t)$ and so

$$
V_{j, \mathbf{F}}^{n, s}=E\left[U_{j^{\prime},\langle x-s . \widetilde{\ell}\rangle}^{n+k} \mid S\left(n \Delta t^{+}\right)=\alpha(s) S_{j}\right] .
$$

In general, $\alpha(s) S_{j}$ does not fall onto the pre-set discrete set of nodal asset values, so we need to perform the quadratic interpolation procedure again for the asset value $\alpha(s) S_{j}$ at time step $n$. The details of the interpolation procedure are illustrated in Figure 2.

The dependency of $V_{j, \mathbf{F}=\langle\mathbf{x}, \ell\rangle}^{n, s}$ on the nodal values computed at a later time level $n+k$ (for $k \geq 1$ ) are revealed by Eqs. (8) and (10). It follows that $U_{0, \mathbf{F}=\left\langle\mathbf{n}_{0}, \mathbf{0}\right\rangle}^{0}$ can 
be computed by backward induction starting at time level $N$. At the end of the liquidation period, we have

$$
U_{j,\langle x, \ell\rangle}^{N}=c(\ell)
$$

where the terminal value depends only on the cash amount.

In summary, suppose the trader at the current time holds a portfolio composition of $x$ units of risky asset and cash that is worth $c^{n}$, he now places a selling order of $s$ units of asset in the market. To compute the maximum expected terminal cash value for this action, we first compute $U_{j^{\prime},\langle x-s, \tilde{\ell}\rangle}^{n+k}$ by quadratic interpolation for cash amount $c^{n+k}$ [see (Eq. (9)], where $k \Delta t=\Delta(s)$ represents the execution time lag for this sell order. We then perform backward induction from these nodal values at time level $n+k$ to time level $n$. After backward induction procedure is completed, we perform interpolation again for the asset price $\alpha(s) S_{j}$ as a result of the instantaneous price drop due to the sell order.

4. Numerical results. The parameter values used in our calculations (unless otherwise specified) are listed in Table 1. In Figure 3, we show the plot of the percentage loss of the liquidation process against the price drop parameter $a$ at varying level of initial number of units of risky asset $n_{0}$ and liquidation time period $T$. The percentage loss of the liquidation process is defined by

$$
\frac{n_{0} S_{0}-E\left(c_{T}\right)}{n_{0} S_{0}} \times 100 \%
$$

where $E\left(c_{T}\right)$ represents the maximum expected terminal value of cash following the optimal liquidation strategy. As expected, the percentage loss is shown to be higher when the price drop parameter $a$ is larger. Moreover, due to the cumulative nature of the price drop effect embedded in the model, the percentage loss is seen to be larger when the initial number of units of risky asset is larger.

We also compute the probability of meeting the target cash level at the end of the liqudation period at different levels $D$ and price drop parameter values $a$. To compute such probability, we simply modify the terminal payoff condition to the following form

$$
U_{j,\langle x, \ell\rangle}^{N}= \begin{cases}1 & \text { if } c(\ell)-D \geq 0 \\ 0 & \text { otherwise }\end{cases}
$$

The algorithm seeks for an optimal trading strategy that maximizes the probability of meeting the target cash level $D$.

From the plots in Figure 4, our numerical results agree with the intuition that the probability decreases as the target level $D$ increases, and the probability becomes lower when the price impact is higher.

We also compare the probability of meeting the target against target cash level with varying values of stock price volatility. The parameter $a$ is chosen to be 0.015 . At a higher target level, our calculations show that the probability becomes higher when the asset price volatility assumes a higher value. On the contrary, at a lower target level, it is seen that the probability becomes smaller when the volatility of the asset price is larger (see Figure 5).

5. Conclusion. Execution time lag exists since a larger sale size requires a longer time to execute the selling order. The "quantity" effect on the asset price is characterized by the liquidation discount, which refers to the difference between the market value of the asset and its value when liquidated. Under the assumption of 


\begin{tabular}{|l|l|}
\hline initial asset price, $S_{0}$ & 100 \\
\hline annualized volatility, $\sigma$ & $30 \%$ \\
\hline annualized drift rate, $\mu$ & $5 \%$ \\
\hline riskless interest rate, $r$ & flat at $2 \%$ per annum \\
\hline initial number of units of asset, $n_{0}$ & 50 \\
\hline liquidation period, $T$ & 5 days \\
\hline execution time lag function, $\Delta(s)$ & $0.1 \times s$ (days) \\
\hline instantaneous price drop function, $\alpha(s)$ & $\frac{0.5}{1-0.5 e^{-a s}}$ \\
\hline instantaneous price drop parameter, $a$ & 0.02 \\
\hline number of time steps used, $N$ & 10 per day \\
\hline
\end{tabular}

TABLE 1. List of parameter values used in our sample calculations.

deterministic execution time lags and liquidation discount, we present a numerical algorithm for computing the optimal liquidation strategy which maximizes the expected terminal value of cash position in a trading portfolio. Such liquidation problem is related to those faced by traders who may need to meet some target cash payment by liquidating the asset in his portfolio. Our algorithm also computes the probability of terminal cash level being above the target.

Our numerical results indicate that the percentage loss of the liquidation process depends significantly on the choice of the price impact function. When the price impact is larger, the percentage loss is shown to be more significant. Furthermore, based on the assumption that the price impact is permanent and cumulative, the percentage loss increases considerably with the number of units of asset in the portfolio. In addition, the probability of meeting the target level is seen to be smaller when the target level and price impact are larger. Depending on the target level, the asset price volatility can have different effects on the probability of meeting the target. When the target level is relatively high, the probability becomes higher when the asset price becomes more volatile. On the contrary, when the target level is relatively low, the probability becomes smaller when the asset price volatility is high. This result seems to differ from other results in the literature that the expected value of the terminal cash position is relatively insensitive to asset price volatility.

\section{REFERENCES}

[1] R.F. Almgren, Optimal execution with nonlinear impact functions and trading-enhanced risk. Applied Mathematical Finance, 10 (2003), 1-18.

[2] R. Almgren, N. Chriss, Optimal execution of portfolio transactions, Journal of Risk, 3(2) (2000), 1-30.

[3] D. Bertsimas, P., Hummer, A.W. Lo, Optimal control of execution costs for portfolios, Computing in Science and Engineering, 1 (2000), 40-53.

[4] D. Bertsimas, A.W. Lo, Optimal control of execution costs, Journal of Financial Markets, 1 (1998), 1-50.

[5] D. Duffie, A. Ziegler, Liquidation risk, Financial Analysts Journal, May/June issue, (2003), $42-51$.

[6] Y.K. Kwok, K.W. Lau, Pricing algorithms for options with exotic path dependence, Journal of Derivatives (Fall, 2001), 28-38.

[7] F.A. Longstaff, Optimal portfolio choice and the valuation of illiquid securities, Review of Financial Studies, 14(2) (2001), 407-431. 
[8] A. Subramanian, R.A. Jarrow The liquidity discount, Mathematical Finance, 11(4) (2001), $447-474$.

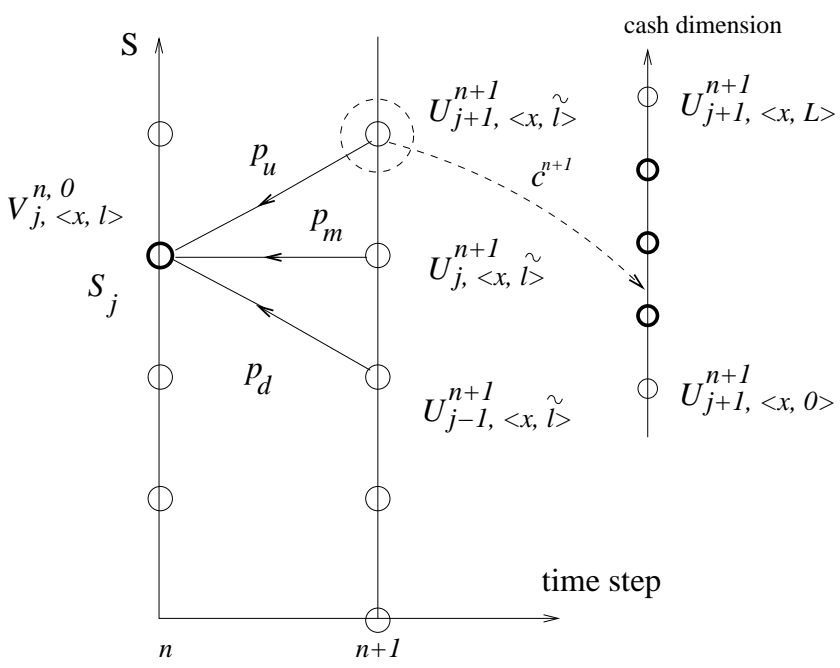

Figure 1. When there is no sale of risky asset, we compute $U_{j+1,<x, \tilde{\ell}>}^{n+1}$ by employing the quadratic interpolation of $c^{n+1}$ at the $(n+1)^{\text {th }}$ time level. 


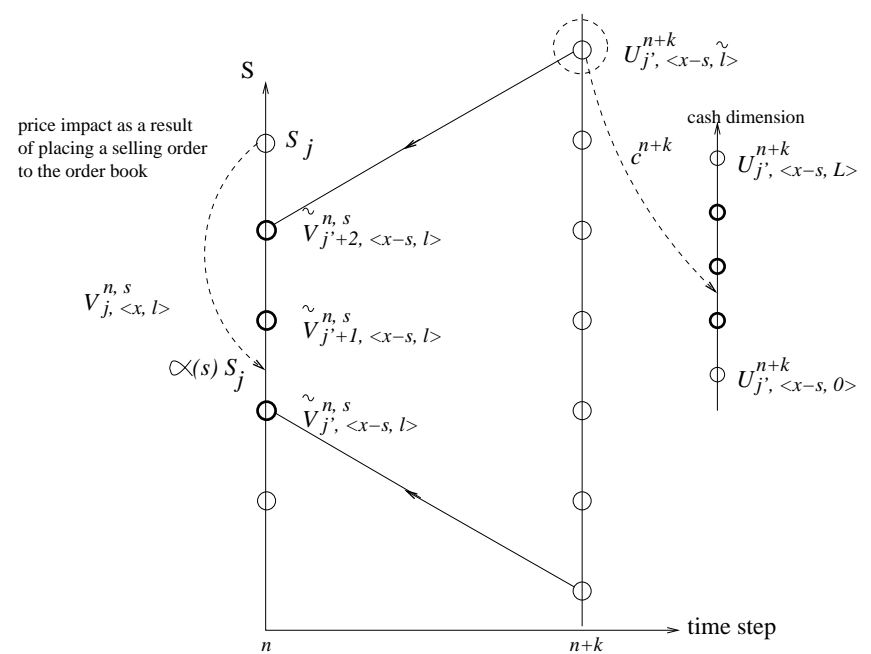

FIGURE 2. Illustration of the backward induction calculations from the $(n+k)^{\text {th }}$ time level to the $n^{\text {th }}$ time level and the implementation of the quadratic interpolation procedure for the cash variable $c^{n+k}$.

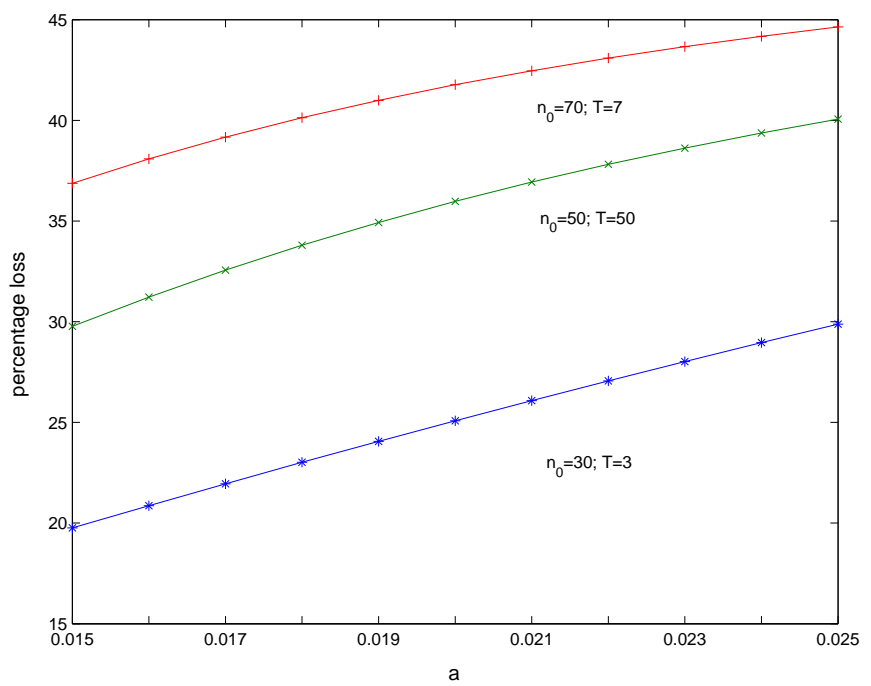

FIGURE 3. Comparison of the percentage loss at varying values of initial number of units of risky asset $n_{0}$, liquidation time period $T$ and price drop parameter $a$. 


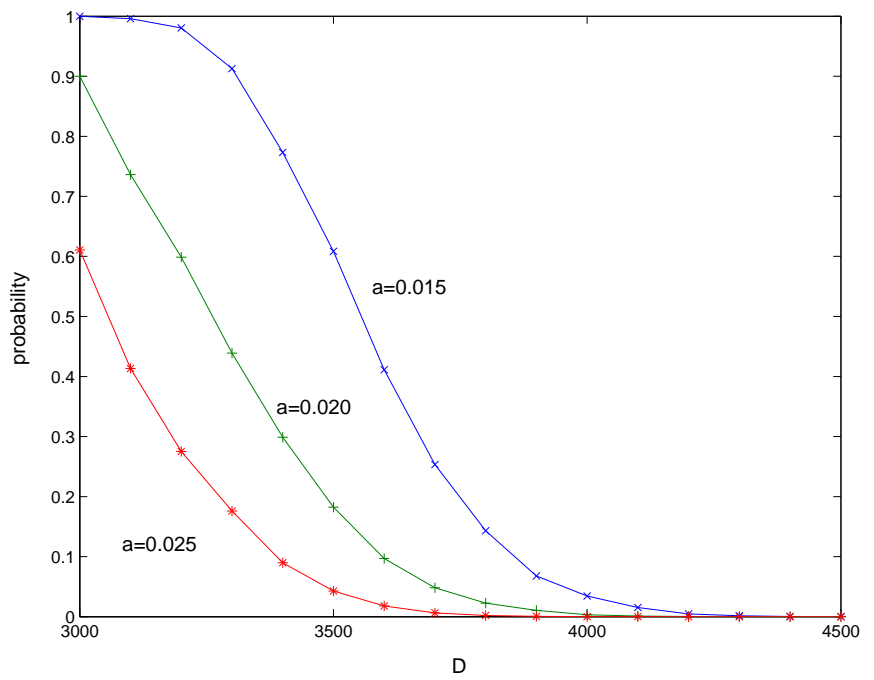

FiguRE 4. Comparison of the probability of terminal cash level above the target level with varying target values $D$ and price drop parameter $a$.

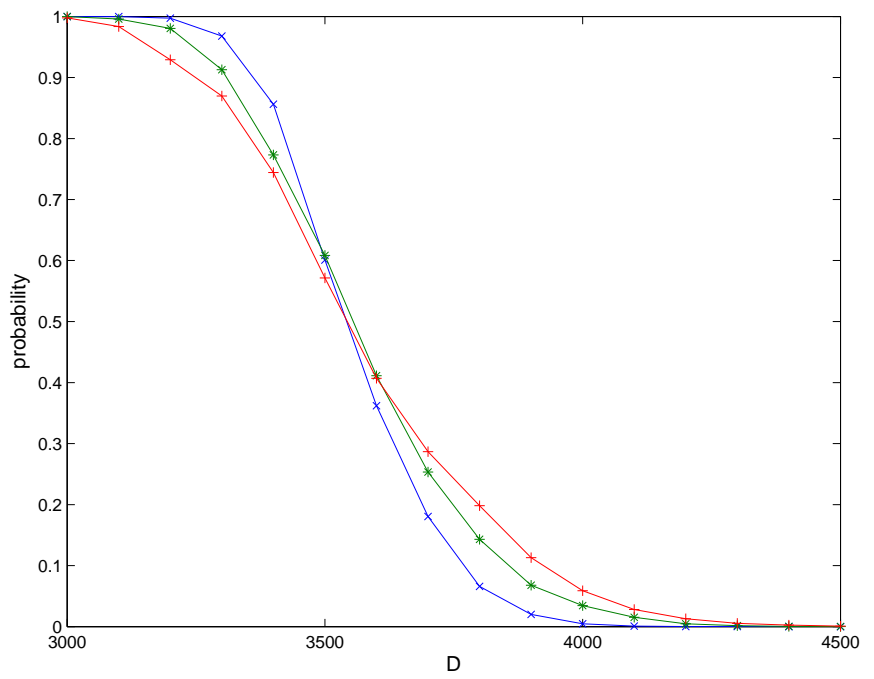

Figure 5. Comparison of the probability of terminal cash level above the target level with varying target values $D$ and asset price volatility level $\sigma$. The volatility values corresponding to the ' $\times$ 'curve, '*'- curve and '+'-curve are $20 \%, 30 \%$ and $40 \%$, respectively. 